\title{
The course and delivery of a pregnancy in a patient with autoimmune hepatitis complicated by cirrhosis of the liver
}

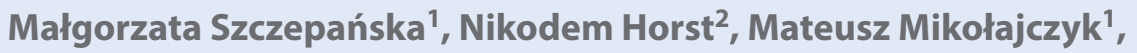 \\ Tomasz Banasiewicz ${ }^{3}$, Ewa Wender-Ożegowska ${ }^{1}$ \\ ${ }^{1}$ Division of Reproduction, Department of Obstetrics, Gynaecology and Gynaecological Oncology, \\ Poznan University of Medical Sciences, Poznan, Poland \\ ${ }^{2}$ Department of General and Colorectal Surgery, Poznan University of Medical Sciences, Poland \\ ${ }^{3}$ Department of General Surgery, Gastroenterological Oncology Surgery and Plastic Surgery, \\ Poznan University of Medical Sciences, Poland
}

We present a case of 28 year old female patient who became pregnant during the qualification for liver transplantation due to decompensated cirrhosis of the liver caused by autoimmune hepatitis (AlH). She was diagnosed at the age of 22 on the basis of the clinical symptoms, serological tests (hypergammaglobulinemia, positive ANA) and microscopic evaluation of the liver (piecemeal necrosis). During this period, there were no indications for liver transplantation. The degree of functional reserve according to the Child-Pugh score was rated as high (class A). The patient was also diagnosed with portal hypertension, which was manifested by splenomegaly and advanced varices of the esophagus and fundus of the stomach. For six years, this patient was first treated with Encorton alone and then with Azathioprine. During this time, she underwent multiple sclerotherapy treatments and endoscopic varices ligation of the esophagus and fundus of the stomach. Six years after being diagnosed with $\mathrm{AlH}$, because of decompensation of the cirrhosis of the liver, manifesting with portal hypertension and symptoms of encephalopathy, indications for liver transplantation were met. During the qualification process, the patient became pregnant. Therefore, the current treatment was changed, Azathioprine was discontinued and the dose of Encorton was increased.

The patient was admitted to the hospital in the $21^{\text {st }}$ week of pregnancy due to vaginal bleeding. During the ultrasound examination, the membranes were detached from the uterus above the internal os of the cervix and the hyperechogenic focus was found, measuring $6.8 \mathrm{~cm}$ in diameter that could correspond to a hematoma. Lutein was administered at a dose of $2 \times 100$ vaginally and Aspargin $4 \times 1$ tablet orally. The abdominal ultrasound confirmed splenomegaly, spleen size in the long axis was approximately $30 \mathrm{~cm}$, it reached with, its lower pole to the bottom of the left hip, and the liver was small with echo structure corresponding to cirrhosis. The ultrasound examination of the fetus confirmed its normal development, consistent with gestational age. Metypred was used at a dose of $24 \mathrm{mg} /$ day for treatment of primary disease. Laboratory tests revealed pancytopenia (Er-2.65 × 10\% $/ \mathrm{mL}$, Leu-2.3 $\times 10^{3} / \mathrm{mL}$, PLT-30 × 10 $3 / \mathrm{mL}, \mathrm{HCT}-0.27 \mathrm{~L} / \mathrm{L}$ ) hyperbilirubinemia ( $3.77 \mathrm{mg} / \mathrm{dL}$ ), elevated bile acid $68.6 \mathrm{micromol} / \mathrm{L}$ and the normal level of transaminases and prolonged prothrombin time (24.4 s) and INR (1.97), decreased prothrombin index of $42 \%$. During the hospitalization, the patient reported haemoptysis and epistaxis.

In the $27^{\text {th }}$ week of pregnancy gestational diabetes was diagnosed, probably due to steroid therapy, and intensive insulin therapy was initiated, resulting in normal glycemic control. Gastroscopy was performed, during which esophageal varices were found in the OMED I stage, without the need for treatment. Due to the signs of preterm labor, betamethasone was used to stimulate fetal lung development. 
At 28 weeks +3 days of gestation, the caesarean section was performed due to acute symptoms fetal distress. A daughter was born with a mass of $1300 \mathrm{~g}, \mathrm{Ap}-1,5,6, \mathrm{pH}$ from umbilical vessels was 7,285 and 7,143. The surgery was performed using non-traumatic threads and preparations increasing local hemostasis (4DryField, TachoSil). Two units of red blood cell concentrate and 11 units of cryoprecipitate were transfused during the postoperative course. In the puerperium, low molecular weight heparin was used in the prophylactic dose and Metypred $24 \mathrm{mg}$. The patient was released home on the $8^{\text {th }}$ day after the Caesarean section. A daughter weighing $3.0 \mathrm{~kg}$ was discharged after 62 days of hospitalization from the Neonatology Clinic.

$\mathrm{AlH}$, especially in a setting of decompensation of the liver function, is a serious complication of pregnancy with a possible catastrophic and sudden consequences, both for the mother and the fetus anytime during pregnancy, and should be managed by a multidisciplinary team, comprising of obstetricians, gastroenterologists, surgeons, anesthesiologists and neonatologists. 